\title{
CADASIL, Migraine and Multiple Sclerosis (MS) - The Risk of Misdiagnosis, Case Report
}

\author{
Piotr Bogucki*1, Paulina Felczak², Teresa Wierzba-Bobrowicz ${ }^{2}$, Halina Sienkiewicz-Jarosz ${ }^{3}$ and Urszula Fiszer ${ }^{1}$ \\ ${ }^{1}$ Department of Neurology and Epileptology, Centre of Postgraduate Medical Education, Poland \\ ${ }^{2}$ Neuropathological Institute, Institute of Psychiatry and Neurology, Poland \\ ${ }^{3}$ First Department of Neurology, Institute of Psychiatry and Neurology, Poland
}

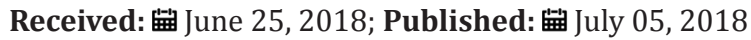

*Corresponding author: Piotr Bogucki, Department of Neurology and Epileptology, Centre of Postgraduate Medical Education, Warsaw, Poland

\begin{abstract}
Diagnostic criteria for multiple sclerosis (MS) have been changing for years to enable easier and faster ways to confirm diagnosis especially during last decade. They lead to earlier treatment of patients with MS what gives higher likelihood to keep patients fit and capable of working. Dissemination in time (DIT) and in space (DIS) are general rules which are necessary to diagnose MS what was maintained in all diagnostic criteria, which have been published up till now[1]. Current criteria were published in 2017 and enable diagnosing MS even in patients, who earlier could not have MS diagnosed. This results from the facts that CSF oligoclonal bands present in patients with DIS without DIT are enough to MS diagnose[2]. Cerebral Autosomal Dominant Arteriopathy with Subcortical Infarcts and Leukoencephalopathy (CADASIL) is a disease of small vessels related to gene NOTCH3 mutations leading to symptoms of migraine with aura, recurrent ischemic incidences, cognitive impairment and behavioral disturbance.
\end{abstract}

Keywords: MS: Multiple Sclerosis; DIT: Dissemination in Time; DIS: Dissemination in Space; CT: Computed Tomography; MRI: Magnetic Resonance Imaging; TPO: Thyroid Peroxidase Antibody; TG: anti-Thyroglobulin Antibody; CADASIL: Cerebral Autosomal Dominant Arteriopathy with Subcortical Infarcts and Leukoencephalopathy; GOM: Granular Osmophilic Material

\section{Case Report}

In this paper we describe patient forty-one years old woman in October of 2012, who was hospitalized in Neurological Ward due to headache, dizziness and numbness of left extremities. Physical examination showed excessive deep reflexes in all extremities and Babinski sign present bilaterally. Computed tomography (CT) revealed multifocal lesions placed subcortically and periventricular. Magnetic resonance imaging (MRI) enabled to visualize numerous hyper intensive lesions in $\mathrm{T}_{2}$-weighted projection, situated in white mater of both hemispheres, peri ventricularly, corpus callous lobe, semi ovalcentres. There were no lesions subtentorially located or enhancing after contrast injection.After that, cerebrospinal fluid was tested - cytosis $1 / \mu \mathrm{L}$, protein $20,6 \mathrm{mg} / \mathrm{dl}$, glucose $62,7 \mathrm{mg} / \mathrm{dl}$ and oligoclonal bands were absent.Therefore, at that moment, MS diagnose was established. Patient was treated using Solu-Medrol following which health ailments neceded. Two months later, in December of 2012, MRI scan of the cervical spinal cord did not reveal any lesions.After two years, in March of 2014, right-sided paraesthesia appeared with concentration disturbances whereas physical examination revealed excessive deep reflexes.

Anti-borrelia antibodies in serum were not revealed. Repeated MRI scan showed similar pictures as before, neither new lesions nor lesions with contrast enhancement, although with the fact, that
U-fibers were not attached suggested to consider whether lesions could not have been resulted from congenital or inflammatory arteriopathy. Diagnose of MS was maintained. In December of 2015, her sister was admitted to the hospital due to speech disturbances, paraesthesia of right upper limb and right side of the face lasting fifteen minutes, followed by a headache. Headaches had been returning for a few years once a week, approximately in anamnesis. Additional tests excluded cardiac source of microembolism. The tests into autoaggressive diseases only thyroid peroxidase antibody (TPO) and antithyroglobulin antibody (TG) were present - TPO $595,0 \mathrm{U} / \mathrm{ml}$, TG 112,3U/ml. MRI scan showed no progression in comparison to the MRI from 2012 (Figures 1-3).

Sister of patient with MS suspicion had similar symptoms and picture of MRI, however she was diagnosed and treated in a different Medical Centre. Therefore, she was diagnosed as a patient with migraine.Considering medical history, familial anamnesis, clinical course of the disease and results of additional tests advanced suspicion of CADASIL. Thus, patient with sister were directed to Institute Psychiatry and Neurology in Warsaw to perform myo-cutaneous biopsy. In this test presence of granular osmophilicmaterial (GOM) was confirmed in patient and her sister [3](Figures 4 \& 5). It enabled to confirm CADASIL diagnose in both 
patient and her sister[4]and [5]. Unfortunately, patients did not agree to a genetic test.

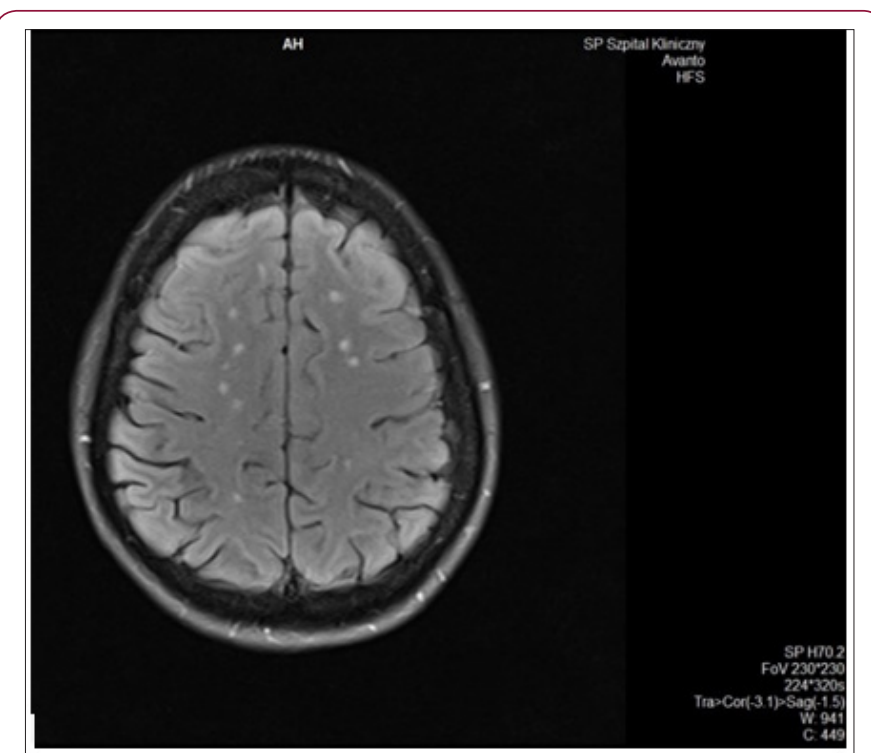

Figure 1

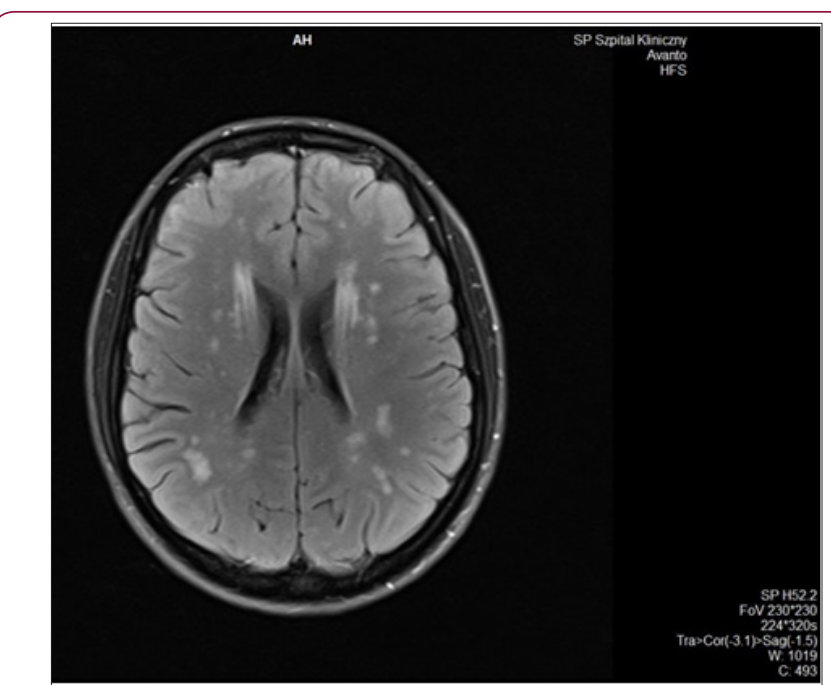

Figure 2

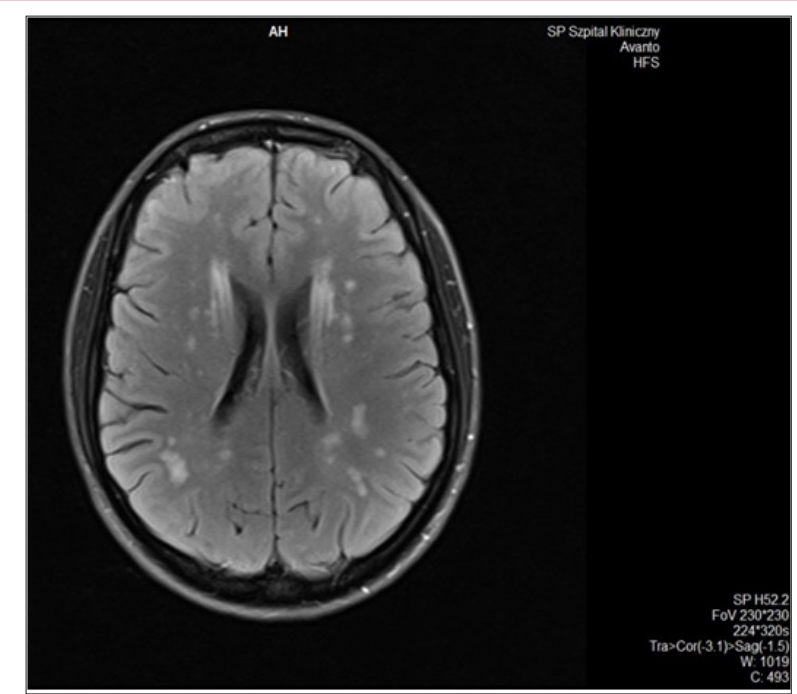

Figure 3

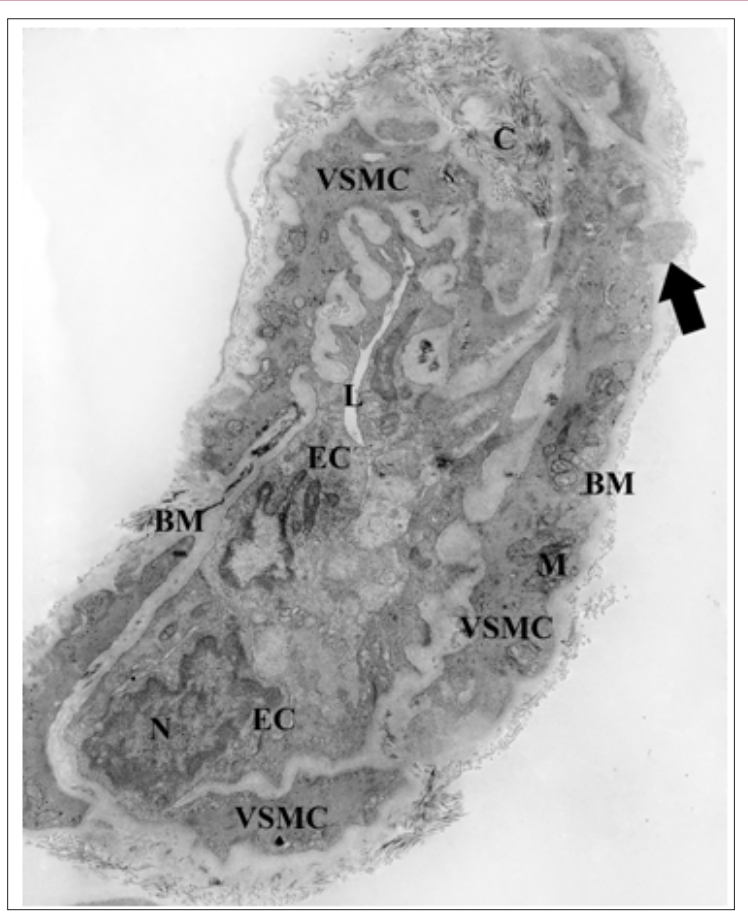

Figure 4: Arteriole. Granular osmiophilic material deposits (GOM) (arrow) located within the basement membrane (BM) near vascular smooth muscle cell (VSMC). Endothelial cell (EC) with nucleus (N). Mitochondria (M), lumen (L), clusters of collagen (C). Orig. magn. 4400x

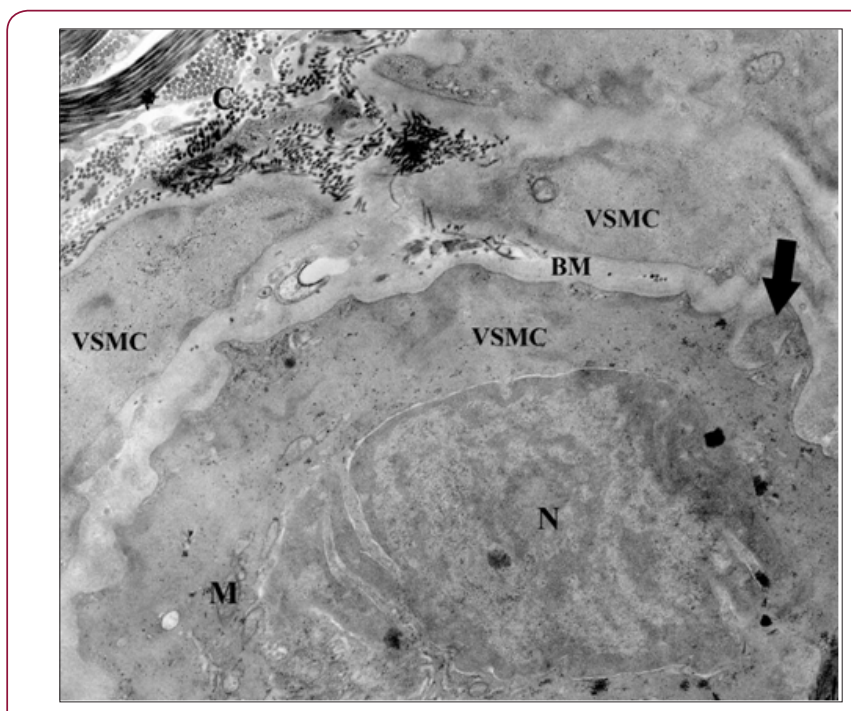

Figure 5: Fragment of arteriole with granular osmiophilic material deposit (GOM) (arrow). Vascular smooth muscle cell (VSMC) with nucleus (N) and mitochondria (M) in cytoplasm. Basement membrane (BM), clusters of collagen (C) Orig. magn. 7000x.

\section{Discussion}

Differential diagnose of MS sometimes can be difficult, especially when clinical course and results of additional test suggest MS diagnose. In this case, MS diagnose was suggested according to MRI results with presence of disseminated lesions, despite the fact that U-fibers were spared. CADASIL is a rare congenital disease which can mimic MS in some cases. Similar misdiagnosis was described 
earlier [6]. According to the new diagnostic criteria of MS which enable earlier diagnose patients with MS another diagnosis should be taken into careful consideration.

\section{References}

1. CH Polman, Reingold SC, Banwell B, Clanet M, Cohen JA, et al. (2011) Diagnostic criteria for multiple sclerosis: 2010 Revisions to the McDonald criteria. Ann Neurol 69(2): 292-302.

2. AJ Thompson, Banwell BL, Barkhof F, Carroll WM, Coetzee T, et al. (2018) Diagnosis of multiple sclerosis: 2017 revisions of the McDonald criteria. Lancet Neurol 17(2): 162-173.

3. T Lorenzi, M Ragno, F Paolinelli, C Castellucci, M Scarpelli, et al. (2017) CADASIL: Ultrastructural insights into the morphology of granular osmiophilic material. Brain Behav 7(3): e00624.

\section{ISSN: 2574-1241}

DOI: $10.26717 / B J S T R .2018 .06 .001344$

Piotr Bogucki. Biomed J Sci \& Tech Res

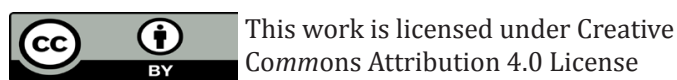

Submission Link: https://biomedres.us/submit-manuscript.php
4. Y Yamamoto, Craggs LJ, Watanabe A, Booth T, Attems J, et al. (2013) Brain MicrovascularAccumulation and Distribution of the NOTCH3 Ectodomain and Granular Osmiophilic Material in CADASIL. J Neuropathol Exp. Neurol 72(5): 416-431.

5. L Chimelli (2017) The role of biopsies and autopsies in the diagnosis of cognitive impairment, with emphasis on small vessel diseases: A critical appraisal enriched by personal experience. Dement Neuropsychol 11(4): 356-363.

6. S Joshi, W Yau, A Kermode (2017) CADASIL mimicking multiple sclerosis: The importance of clinical and MRI red flags. J Clinical Neuroscience 35: 75-77.

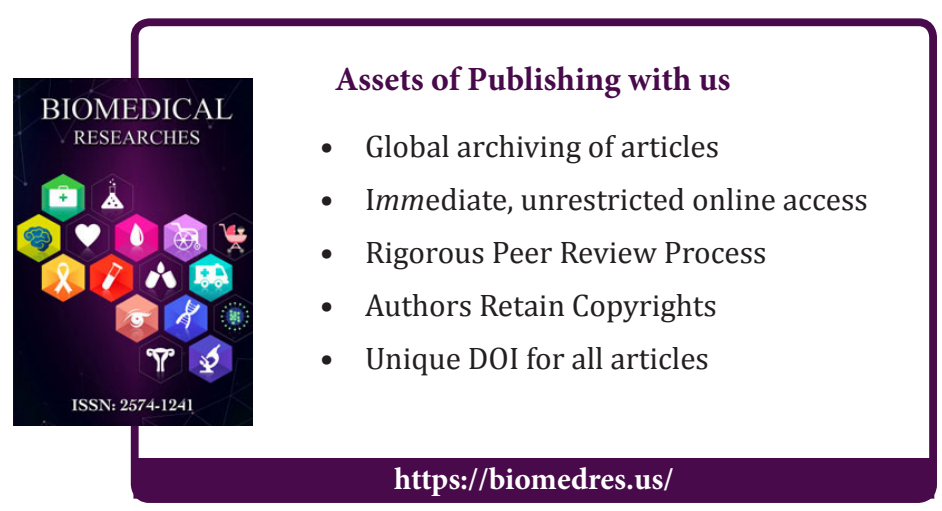

